\title{
A Strong Limit Theorem for Functions of Continuous Random Variables and an Extension of the Shannon-McMillan Theorem
}

\author{
Gaorong Li, ${ }^{1,2}$ Shuang Chen, ${ }^{3}$ and Sanying Feng ${ }^{4}$ \\ ${ }^{1}$ School of Finance and Statistics, East China Normal University, Shanghai 200241, China \\ ${ }^{2}$ College of Applied Sciences, Beijing University of Technology, Beijing 100022, China \\ ${ }^{3}$ School of Sciences, Hebei University of Technology, Tianjin 300130, China \\ ${ }^{4}$ College of Mathematics and Science, Luoyang Normal University, Henan 471022, China
}

Correspondence should be addressed to Gaorong Li, 1_gaorong@163.com

Received 1 November 2007; Revised 10 January 2008; Accepted 29 March 2008

Recommended by Onno Boxma

By means of the notion of likelihood ratio, the limit properties of the sequences of arbitrarydependent continuous random variables are studied, and a kind of strong limit theorems represented by inequalities with random bounds for functions of continuous random variables is established. The Shannon-McMillan theorem is extended to the case of arbitrary continuous information sources. In the proof, an analytic technique, the tools of Laplace transform, and moment generating functions to study the strong limit theorems are applied.

Copyright (C) 2008 Gaorong Li et al. This is an open access article distributed under the Creative Commons Attribution License, which permits unrestricted use, distribution, and reproduction in any medium, provided the original work is properly cited.

\section{Introduction}

Let $\left\{X_{n}, n \geq 1\right\}$ be a sequence of arbitrary continuous real random variables on the probability space $(\Omega, \mp, P)$ with the joint density function

$$
f_{n}\left(x_{1}, \ldots, x_{n}\right)>0, \quad n=1,2, \ldots,
$$

where $x_{i} \in(-\infty, \infty), 1 \leq i \leq n$. Let $Q$ be another probability measure on $\mathcal{F}$, and $\left\{X_{n}, n \geq 1\right\}$ is a sequence of independent random variables on the probability space $(\Omega, \mathcal{F}, Q)$ with the marginal density functions $g_{k}\left(x_{k}\right)(1 \leq k \leq n)$, and let

$$
\pi_{n}\left(x_{1}, \ldots, x_{n}\right)=\prod_{k=1}^{n} g_{k}\left(x_{k}\right) .
$$


In order to indicate the deviation between $\left\{X_{n}, n \geq 1\right\}$ on the probability measure $P$ and $Q$, we first introduce the following definitions.

Definition 1.1. Let $\left\{X_{n}, n \geq 1\right\}$ be a sequence of random variables with joint distribution (1.1), and let $g_{k}\left(x_{k}\right)(k=1,2, \ldots, n)$ be defined by (1.2). Let

$$
Z_{n}(\omega)=\frac{f_{n}\left(X_{1}, \ldots, X_{n}\right)}{\pi_{n}\left(X_{1}, \ldots, X_{n}\right)}
$$

In statistical terms, $Z_{n}(\omega)$ is called the likelihood ratio, which is of fundamental importance in the theory of testing the statistical hypotheses (cf. [1, page 388]; [2, page 483]).

The random variable

$$
r(\omega)=\limsup _{n \rightarrow \infty} \frac{1}{n} \ln Z_{n}(\omega)=-\liminf _{n \rightarrow \infty} \frac{1}{n} \ln \left[\left\{\prod_{k=1}^{n} g_{k}\left(X_{k}\right)\right\} / f_{n}\left(X_{1}, \ldots, X_{n}\right)\right]
$$

is called asymptotic logarithmic likelihood ratio, relative to the product of marginal distribution of (1.2), of $X_{n}, n \geq 1$, where $\ln$ is the natural logarithm, $\omega$ is the sample point. For the sake of brevity, we denote $X_{k}(\omega)$ by $X_{k}$.

Although $r(\omega)$ is not a proper metric between probability measures, we nevertheless think of it as a measure of "dissimilarity" between their joint distribution $f_{n}\left(x_{1}, \ldots, x_{n}\right)$ and the product $\pi_{n}\left(x_{1}, \ldots, x_{n}\right)$ of their marginals.

Obviously, $r(\omega)=0$, a.s. if and only if $\left\{X_{n}, n \geq 1\right\}$ are independent.

A stochastic process of fundamental importance in the theory of testing hypotheses is the sequence of likelihood ratio. In view of the above discussion of the asymptotic logarithmic likelihood ratio, it is natural to think of $r(\omega)$ as a measure how far (the random deviation of) $X_{n}$ is from being independent, how dependent they are. The smaller $r(\omega)$ is, the smaller the deviation is (cf. [3-5]).

In [3], the strong deviation theorems for discrete random variables were discussed by using the generating function method. Later, the approach of Laplace transform to study the strong limit theorems was first proposed by Liu [4]. Yang [6] further studied the limit properties for Markov chains indexed by a homogeneous tree through the analytic technique. Many comprehensive works may be found in Liu [7]. The purpose of this paper is to establish a kind of strong deviation theorems represented by inequalities with random bounds for functions of arbitrary continuous random variables, by combining the analytic technique with the method of Laplace transform, and to extend the strong deviation theorems to the differential entropy for arbitrary-dependent continuous information sources in more general settings.

Definition 1.2. Let $\left\{h_{n}\left(x_{n}\right), n \geq 1\right\}$ be a sequence of nonnegative $B$ orel measurable functions defined on $R$, the Laplace transform of random variables $h_{n}\left(X_{n}\right)$ on the probability space $(\Omega, \mathcal{F}, Q)$ is defined by

$$
\tilde{f}_{n}(s)=\int_{-\infty}^{\infty} e^{-s h_{n}\left(x_{n}\right)} g_{n}\left(x_{n}\right) d x_{n}=E_{Q} e^{-s h_{n}\left(X_{n}\right)}, \quad n=1,2, \ldots,
$$

where $E_{Q}$ denotes the expectation under $Q$. 
We have the following assumptions in this paper.

(1) Assume that there exists $s_{0} \in(0, \infty)$ such that

$$
\tilde{f}_{n}(s)<\infty, \quad s \in\left[-s_{0}, s_{0}\right], n=1,2, \ldots
$$

(2) Assume $M>0$ is a constant, satisfying

$$
\sup _{n} E_{Q} h_{n}\left(X_{n}\right) \leq M<\infty .
$$

In order to prove our main results, we first give a lemma, and it will be shown that it plays a central role in the proofs.

Lemma 1.3. Let $f_{n}\left(x_{1}, \ldots, x_{n}\right), g_{n}\left(x_{1}, \ldots, x_{n}\right)$ be two probability functions on $(\Omega, \mathcal{F}, P)$, let

$$
T_{n}(\omega)=\frac{g_{n}\left(X_{1}, \ldots, X_{n}\right)}{f_{n}\left(X_{1}, \ldots, X_{n}\right)}
$$

then

$$
\limsup _{n \rightarrow \infty} \frac{1}{n} \ln T_{n}(\omega) \leq 0 \quad \text { a.s. }
$$

Proof. By [8], $\left\{T_{n}, \mathcal{F}, n \geq 1\right\}$ is a nonnegative martingale and $E T_{n}=1$, we have by the Doob martingale convergence theorem, there exists an integral random variable $T_{\infty}(\omega)$, such that $T_{n} \rightarrow T_{\infty}$, a.s. and (1.9) follows.

\section{Main results}

Theorem 2.1. Let $\left\{X_{n}, n \geq 1\right\}, Z_{n}(\omega), r(\omega), \tilde{f}_{n}(s)$ be defined as before, and under the assumptions of (1) and (2), let

$$
\limsup _{n \rightarrow \infty} \frac{1}{n} \sum_{k=1}^{n} E_{Q} e^{s_{0} h_{k}\left(X_{k}\right)}=\limsup _{n \rightarrow \infty} \frac{1}{n} \sum_{k=1}^{n} \tilde{f}_{k}\left(-s_{0}\right)=M\left(s_{0}\right)<\infty .
$$

Then

$$
\begin{aligned}
& \liminf _{n \rightarrow \infty} \frac{1}{n} \sum_{k=1}^{n}\left[h_{k}\left(X_{k}\right)-E_{Q} h_{k}\left(X_{k}\right)\right] \geq-\beta(r(\omega)), \quad \text { a.s. } \\
& \limsup _{n \rightarrow \infty} \frac{1}{n} \sum_{k=1}^{n}\left[h_{k}\left(X_{k}\right)-E_{Q} h_{k}\left(X_{k}\right)\right] \leq \beta(r(\omega)), \quad \text { a.s. }
\end{aligned}
$$

where

$$
\begin{gathered}
\beta(x)=\inf \left\{\phi(s, x),-s_{0}<s<0\right\}, \quad x \geq 0, \\
\phi(s, x)=-\frac{2 e^{-2} s M\left(s_{0}\right)}{\left(s_{0}-|s|\right)^{2}}-\frac{x}{s}, \quad x \geq 0, \\
0 \leq \beta(x) \leq \frac{\sqrt{x}(1+\sqrt{x})}{s_{0}}\left[2 e^{-2} M\left(s_{0}\right)+1\right], \quad \lim _{x \rightarrow 0+} \beta(x)=\beta(0)=0 .
\end{gathered}
$$


Remark 2.2. Let

$$
\alpha(x)=\sup \left\{\phi(s, x), 0<s<s_{0}\right\}, \quad x \geq 0,
$$

then

$$
\alpha(x)=-\beta(x) .
$$

Proof. Let $s$ be an arbitrary real number in $\left(-s_{0}, s_{0}\right)$, let

$$
g_{n}\left(s, x_{n}\right)=e^{-s h_{n}\left(x_{n}\right)} g_{n}\left(x_{n}\right) / \tilde{f}_{n}(s), \quad x_{n} \in(-\infty, \infty), n=1,2, \ldots,
$$

then $\int_{-\infty}^{\infty} g_{n}\left(s, x_{n}\right) d x_{n}=1$, and let

$$
q_{n}\left(s ; x_{1}, \ldots, x_{n}\right)=\prod_{k=1}^{n} g_{k}\left(s, x_{k}\right)
$$

Therefore, $q_{n}\left(s ; x_{1}, \ldots, x_{n}\right)$ is an $n$ multivariate probability density function, let

$$
t_{n}(s, \omega)=\frac{q_{n}\left(s ; X_{1}, \ldots, X_{n}\right)}{f_{n}\left(X_{1}, \ldots, X_{n}\right)}, \quad n=1,2, \ldots
$$

By Lemma 1.3, there exists a set $A(s)$ such that $P(A(s))=1$, so we have

$$
\limsup _{n \rightarrow \infty} \frac{1}{n} \ln t_{n}(s, \omega) \leq 0, \quad \omega \in A(s) .
$$

By (1.3), (2.9), (2.11), and (2.12), we have

$$
\limsup _{n \rightarrow \infty} \frac{1}{n}\left[-s \sum_{k=1}^{n} h_{k}\left(X_{k}\right)-\sum_{k=1}^{n} \ln \tilde{f}_{k}(s)-\ln Z_{n}(\omega)\right] \leq 0, \quad \omega \in A(s) .
$$

Therefore,

$$
r(\omega) \geq 0, \quad \omega \in A(0) .
$$

By (2.13) and (1.4), the property of the superior limit

$$
\limsup _{n \rightarrow \infty}\left(a_{n}-b_{n}\right) \leq 0 \Longrightarrow \limsup _{n \rightarrow \infty} a_{n} \leq \limsup _{n \rightarrow \infty} b_{n}
$$

and the inequality $\ln x \leq x-1(x>0)$, we have

$$
\begin{aligned}
\limsup _{n \rightarrow \infty} & \frac{1}{n}(-s) \sum_{k=1}^{n}\left[h_{k}\left(X_{k}\right)-E_{Q} h_{k}\left(X_{k}\right)\right] \\
\quad \leq & \limsup _{n \rightarrow \infty} \frac{1}{n} \sum_{k=1}^{n}\left[\ln \tilde{f}_{k}(s)+s E_{Q} h_{k}\left(X_{k}\right)\right]+r(\omega) \\
\quad \leq & \limsup _{n \rightarrow \infty} \frac{1}{n} \sum_{k=1}^{n}\left[\tilde{f}_{k}(s)-1+s E_{Q} h_{k}\left(X_{k}\right)\right]+r(\omega) \\
\quad & \limsup _{n \rightarrow \infty} \frac{1}{n} \sum_{k=1}^{n}\left[\tilde{f}_{k}(s)-1-(-s) E_{Q} h_{k}\left(X_{k}\right)\right]+r(\omega), \quad \omega \in A(s) .
\end{aligned}
$$


By the inequality $0 \leq e^{x}-1-x \leq(1 / 2) x^{2} e^{|x|}$, which can be found in [9], we have

$$
0 \leq e^{-s h_{k}\left(X_{k}\right)}-1-(-s) h_{k}\left(X_{k}\right) \leq(1 / 2)\left(s h_{k}\left(X_{k}\right)\right)^{2} e^{\left|s h_{k}\left(X_{k}\right)\right|}
$$

By (2.5) and (2.17), we have

$$
\begin{aligned}
\limsup _{n \rightarrow \infty} & \frac{1}{n}(-s) \sum_{k=1}^{n}\left[h_{k}\left(X_{k}\right)-E_{Q} h_{k}\left(X_{k}\right)\right] \\
\leq & (1 / 2) s^{2} \limsup _{n \rightarrow \infty} \frac{1}{n} \sum_{k=1}^{n} E_{Q}\left[\left(h_{k}\left(X_{k}\right)\right)^{2} e^{|s| h_{k}\left(X_{k}\right)}\right]+r(\omega), \quad \omega \in A(s) .
\end{aligned}
$$

It is easy to see that $\varphi(x)=t^{x} x^{2}(t>1)$ attains its largest value $\varphi(-2 / \ln t)=4 e^{-2} /(\ln t)^{2}$ on the interval $(-\infty, 0]$, and $\varphi(x)=t^{x} x^{2}(0<t<1)$ attains its largest value $\varphi(-2 / \ln t)=$ $4 e^{-2} /(\ln t)^{2}$ on the interval $[0, \infty)$, we have

$$
\begin{gathered}
\sup \left\{e^{\left(s-s_{0}\right) h_{k}\left(X_{k}\right)}\left[h_{k}\left(X_{k}\right)\right]^{2}, k \geq 1\right\} \leq \frac{4 e^{-2}}{\left(s-s_{0}\right)^{2}}=\frac{4 e^{-2}}{\left(s_{0}-s\right)^{2}}, \quad 0<s<s_{0}, \\
\sup \left\{e^{\left(s_{0}+s\right)\left(-h_{k}\left(X_{k}\right)\right)}\left[-h_{k}\left(X_{k}\right)\right]^{2}, k \geq 1\right\} \leq \frac{4 e^{-2}}{\left(s_{0}+s\right)^{2}}, \quad-s_{0}<s<0 .
\end{gathered}
$$

Let $0<s<s_{0}$ in (2.18), by (2.19) and (2.1), we obtain

$$
\begin{aligned}
(-s) & \liminf _{n \rightarrow \infty} \frac{1}{n} \sum_{k=1}^{n}\left[h_{k}\left(X_{k}\right)-E_{Q} h_{k}\left(X_{k}\right)\right] \\
& \leq(1 / 2) s^{2} \limsup _{n \rightarrow \infty} \frac{1}{n} \sum_{k=1}^{n} E_{Q}\left[\left(h_{k}\left(X_{k}\right)\right)^{2} e^{|s| h_{k}\left(X_{k}\right)}\right]+r(\omega) \\
& =(1 / 2) s^{2} \limsup _{n \rightarrow \infty} \frac{1}{n} \sum_{k=1}^{n} E_{Q}\left[e^{s_{0} h_{k}\left(X_{k}\right)} e^{\left(s-s_{0}\right) h_{k}\left(X_{k}\right)}\left(h_{k}\left(X_{k}\right)\right)^{2}\right]+r(\omega) \\
& \leq \frac{2 e^{-2} s^{2} M\left(s_{0}\right)}{\left(s_{0}-s\right)^{2}}+r(\omega), \quad \omega \in A(s) .
\end{aligned}
$$

Dividing the two sides of (2.21) by $-s$, we obtain

$$
\begin{aligned}
\liminf _{n \rightarrow \infty} \frac{1}{n} \sum_{k=1}^{n}\left[h_{k}\left(X_{k}\right)-E_{Q} h_{k}\left(X_{k}\right)\right] & \geq-\frac{2 e^{-2} s M\left(s_{0}\right)}{\left(s_{0}-s\right)^{2}}-\frac{r(\omega)}{s} \\
& \hat{=} \phi(s, r(\omega)), \quad 0<s<s_{0}, \omega \in A(s) \cap A(0) .
\end{aligned}
$$

By (2.14) and $0<s<s_{0}$, obviously $\phi(s, r(\omega)) \leq 0$, hence $\alpha(r(\omega)) \leq 0$. Let $Q^{+}$be the set of rational numbers in the interval $\left(0, s_{0}\right)$, and let $A^{*}=\cap_{s \in Q^{+}} A(s)$, then $P\left(A^{*}\right)=1$. By (2.22), then we have

$$
\liminf _{n \rightarrow \infty} \frac{1}{n} \sum_{k=1}^{n}\left[h_{k}\left(X_{k}\right)-E_{Q} h_{k}\left(X_{k}\right)\right] \geq \phi(s, r(\omega)), \quad \omega \in A^{*} \cap A(0), \forall s \in Q^{+} .
$$


It is easy to see that $\phi(s, x)$ is a continuous function with respect to $s$ on the interval $\left(0, s_{0}\right)$. For each $\omega \in A^{*} \cap A(0)(0 \leq r(\omega)<\infty)$, take $s_{n}(\omega) \in Q^{+}, n=1,2, \ldots$, such that

$$
\lim _{n \rightarrow \infty} \phi\left(s_{n}(\omega), r(\omega)\right)=\alpha(r(\omega)) .
$$

By (2.23), (2.24), and (2.8), we have

$$
\liminf _{n \rightarrow \infty} \frac{1}{n} \sum_{k=1}^{n}\left[h_{k}\left(X_{k}\right)-E_{Q} h_{k}\left(X_{k}\right)\right] \geq-\beta(r(\omega)), \quad \omega \in A^{*} \cap A(0) .
$$

Since $P\left(A^{*} \cap A(0)\right)=1$, (2.2) follows from (2.25).

Let $-s_{0}<s<0$ in (2.18), by (2.20) and (2.1), we have

$$
\begin{aligned}
\limsup _{n \rightarrow \infty} & \frac{1}{n} \sum_{k=1}^{n}\left[h_{k}\left(X_{k}\right)-E_{Q} h_{k}\left(X_{k}\right)\right] \\
\leq & -(1 / 2) s \limsup _{n \rightarrow \infty} \frac{1}{n} \sum_{k=1}^{n} E_{Q}\left[\left(h_{k}\left(X_{k}\right)\right)^{2} e^{|s| h_{k}\left(X_{k}\right)}\right]-\frac{r(\omega)}{s} \\
& =-(1 / 2) s \limsup _{n \rightarrow \infty} \frac{1}{n} \sum_{k=1}^{n} E_{Q}\left[e^{s_{0} h_{k}\left(X_{k}\right)} e^{\left(s_{0}+s\right)\left(-h_{k}\left(X_{k}\right)\right)}\left(-h_{k}\left(X_{k}\right)\right)^{2}\right]-\frac{r(\omega)}{s} \\
& \leq-\frac{2 e^{-2} s M\left(s_{0}\right)}{\left(s_{0}+s\right)^{2}}-\frac{r(\omega)}{s} \\
& =\phi(s, r(\omega)), \quad-s_{0}<s<0, \omega \in A(s) \cap A(0) .
\end{aligned}
$$

By (2.14) and $-s_{0}<s<0$, obviously $\phi(s, r(\omega)) \geq 0$, hence $\beta(r(\omega)) \geq 0$. Let $Q^{-}$be the set of rational numbers in the interval $\left(-s_{0}, 0\right)$, and let $A_{*}=\cap_{s \in Q^{-}} A(s)$, then $P\left(A_{*}\right)=1$. Then we have by (2.26)

$$
\limsup _{n \rightarrow \infty} \frac{1}{n} \sum_{k=1}^{n}\left[h_{k}\left(X_{k}\right)-E_{Q} h_{k}\left(X_{k}\right)\right] \leq \phi(s, r(\omega)), \quad \omega \in A_{*} \cap A(0), \forall s \in Q^{-} .
$$

It is clear that $\phi(s, x)$ is a continuous function with respect to $s$ on the interval $\left(-s_{0}, 0\right)$. For each $\omega \in A_{*} \cap A(0)(0 \leq r(\omega)<\infty)$, take $\lambda_{n}(\omega) \in Q^{-}, n=1,2, \ldots$, such that

$$
\lim _{n \rightarrow \infty} \phi\left(\lambda_{n}(\omega), r(\omega)\right)=\beta(r(\omega)) .
$$

By (2.27) and (2.28), we have

$$
\limsup _{n \rightarrow \infty} \frac{1}{n} \sum_{k=1}^{n}\left[h_{k}\left(X_{k}\right)-E_{Q} h_{k}\left(X_{k}\right)\right] \leq \beta(r(\omega)), \quad \omega \in A_{*} \cap A(0) .
$$

Since $P\left(A_{*} \cap A(0)\right)=1$, (2.3) follows from (2.29).

By (2.4), (2.5), and (2.14), if $x>0$, we have

$$
0 \leq \beta(x) \leq \phi\left(-\frac{s_{0} \sqrt{x}}{1+\sqrt{x}}, x\right)=\frac{\sqrt{x}(1+\sqrt{x})}{s_{0}}\left[2 e^{-2} M\left(s_{0}\right)+1\right] .
$$

If $x=0$, we have

$$
\beta(0) \leq \phi\left(-n^{-1}, 0\right)=\frac{2 e^{-2} M\left(s_{0}\right)}{n\left(s_{0}-n^{-1}\right)^{2}}, \quad n \geq 1 .
$$

Noticing that $\beta(x) \geq 0,(x \geq 0)$, (2.6) follows from (2.30) and (2.31). 
Corollary 2.3. If $P=Q$, or $\left\{X_{n}, n \geq 1\right\}$ is a sequence of independent random variables, and under the assumptions of (1) and (2), then

$$
\lim _{n \rightarrow \infty} \frac{1}{n} \sum_{k=1}^{n}\left[h_{k}\left(X_{k}\right)-E h_{k}\left(X_{k}\right)\right]=0 \quad \text { a.s. }
$$

Proof. In this case, $f_{n}\left(x_{1}, \ldots, x_{n}\right)=\prod_{k=1}^{n} g_{k}\left(x_{k}\right)$, and $r(\omega)=0$ a.s. Hence, (2.32) follows directly from (2.2) and (2.3).

\section{An extension of the Shannon-McMillan theorem}

In order to understand better, we first introduce some definitions in information theory in this section.

Let $\left\{X_{n}, n \geq 1\right\}$ be a sequence produced by an arbitrary continuous information source on the probability space $(\Omega, \mathcal{F}, P)$ with the joint density function

$$
f_{n}\left(x_{1}, \ldots, x_{n}\right)>0, \quad x_{k} \in(-\infty, \infty), \quad 1 \leq k \leq n, n=1,2, \ldots
$$

For the sake of brevity, we denote $f_{n}>0$, and $X_{k}$ stands for $X_{k}(\omega)$. Let

$$
p_{n}(\omega)=-(1 / n) \ln f_{n}\left(X_{1}, \ldots, X_{n}\right),
$$

where $\omega$ is the sample point, $p_{n}(\omega)$ is called the sample entropy or the entropy density of $\left\{X_{k}, 1 \leq k \leq n\right\}$. Also let $Q$ be another probability measure on $\mp$ with the density function

$$
q_{n}\left(x_{1}, \ldots, x_{n}\right)>0, \quad x_{k} \in(-\infty, \infty), \quad 1 \leq k \leq n, n=1,2, \ldots
$$

Let

$$
\begin{aligned}
L_{n}(\omega) & =\ln \left[f_{n}\left(X_{1}, \ldots, X_{n}\right) / q_{n}\left(X_{1}, \ldots, X_{n}\right)\right], \\
L(\omega) & =\limsup _{n \rightarrow \infty}(1 / n) L_{n}(\omega) \\
& =-\liminf _{n \rightarrow \infty}(1 / n) \ln \left[q_{n}\left(X_{1}, \ldots, X_{n}\right) / f_{n}\left(X_{1}, \ldots, X_{n}\right)\right], \\
D\left(f_{n} \| q_{n}\right) & =E_{P} L_{n} \\
& =E_{P} \ln \left[f_{n}\left(X_{1}, \ldots, X_{n}\right) / q_{n}\left(X_{1}, \ldots, X_{n}\right)\right] .
\end{aligned}
$$

$L_{n}(\omega), L(\omega)$, and $D\left(f_{n} \| q_{n}\right)$ are called the sample relative entropy, the sample relative entropy rate, and the relative entropy, respectively, relative to the reference density function $q_{n}\left(x_{1}, \ldots, x_{n}\right)$. Indeed, they all are the measure of the deviation between the true joint distribution density function $f_{n}\left(x_{1}, \ldots, x_{n}\right)$ and the reference distribution density function $q_{n}\left(x_{1}, \ldots, x_{n}\right)$ (cf. [10, pages 12, 18]).

A question of importance in information theory is the study of the limit properties of the relative entropy density $f_{n}(\omega)$. Since Shannon's initial work was published (cf. [11]), there has been a great deal of investigation about this question (e.g., cf. [12-20]).

In this paper, a class of small deviation theorems (i.e., the strong limit theorems represented by inequalities) is established by using the analytical technique, and an extension of the Shannon-McMillan theorem to the arbitrary-dependent continuous information sources is given. Especially, an approach of applying the tool of Laplace transform to the study of the strong deviation theorems on the differential entropy is proposed. 
Let $h_{k}\left(x_{k}\right)=-\ln g_{k}\left(x_{k}\right)(1 \leq k \leq n, n=1,2, \ldots)$ in (1.5), then we give the following definitions.

Definition 3.1. The Laplace transform of $-\ln g_{k}\left(x_{k}\right)$ is defined by

$$
\tilde{f}_{k}(s)=E e^{-s\left(-\ln g_{k}\left(X_{k}\right)\right)}=\int_{-\infty}^{\infty} e^{-s\left(-\ln g_{k}\left(x_{k}\right)\right)} g_{k}\left(x_{k}\right) d x_{k} .
$$

Definition 3.2. The differential entropy for continuous random variables $X_{k}$ is defined by

$$
h\left(X_{k}\right)=E\left[-\ln g_{k}\left(X_{k}\right)\right]=-\int_{-\infty}^{\infty} g_{k}\left(x_{k}\right) \ln g_{k}\left(x_{k}\right) d x_{k}
$$

In the following theorem, let $\left\{X_{n}, n \geq 1\right\}$ be independent random variables with respect to $Q$, then the reference density function $q_{n}\left(x_{1}, \ldots, x_{n}\right)=\prod_{k=1}^{n} g_{k}\left(x_{k}\right)$, and let $h_{k}\left(X_{k}\right)=$ $-\ln g_{k}\left(X_{k}\right)(1 \leq k \leq n)$ in Theorem 2.1 .

Theorem 3.3. Let $\left\{X_{n}, n \geq 1\right\}, L_{n}(\omega), L(\omega), \tilde{f}_{n}(s)$ be given as above, and under the assumptions of (1) and (2), let

$$
\limsup _{n \rightarrow \infty} \frac{1}{n} \sum_{k=1}^{n} E_{Q} e^{s_{0}\left(-\ln g_{k}\left(X_{k}\right)\right)}=\limsup _{n \rightarrow \infty} \frac{1}{n} \sum_{k=1}^{n} \tilde{f}_{k}\left(-s_{0}\right)=M\left(s_{0}\right)<\infty
$$

Then

$$
\begin{aligned}
& \liminf _{n \rightarrow \infty} \frac{1}{n} \sum_{k=1}^{n}\left[-\ln g_{k}\left(X_{k}\right)-h\left(X_{k}\right)\right] \geq-\beta(L(\omega)), \quad \text { a.s. } \\
& \limsup _{n \rightarrow \infty} \frac{1}{n} \sum_{k=1}^{n}\left[-\ln g_{k}\left(X_{k}\right)-h\left(X_{k}\right)\right] \leq \beta(L(\omega)), \quad \text { a.s. }
\end{aligned}
$$

where

$$
\begin{gathered}
\beta(x)=\inf \left\{\phi(s, x),-s_{0}<s<0\right\}, \quad x \geq 0, \\
\phi(s, x)=-\frac{2 e^{-2} s M\left(s_{0}\right)}{\left(s_{0}-|s|\right)^{2}}-\frac{x}{s}, \quad x \geq 0, \\
0 \leq \beta(x) \leq \frac{\sqrt{x}(1+\sqrt{x})}{s_{0}}\left[2 e^{-2} M\left(s_{0}\right)+1\right], \quad \lim _{x \rightarrow 0+} \beta(x)=\beta(0)=0 .
\end{gathered}
$$

Remark 3.4. Let

$$
\alpha(x)=\sup \left\{\phi(s, x), 0<s<s_{0}\right\}, \quad x \geq 0,
$$

then

$$
\alpha(x)=-\beta(x)
$$


Corollary 3.5. Let $p_{n}(\omega)$ be defined by (3.2). Under the condition of Theorem 3.3, then

$$
\begin{gathered}
\liminf _{n \rightarrow \infty}\left[p_{n}(\omega)-(1 / n) h\left(X_{1}, \ldots, X_{n}\right)\right] \geq \alpha(L(\omega))-L(\omega)+H_{*} \quad \text { a.s. } \\
\limsup _{n \rightarrow \infty}\left[p_{n}(\omega)-(1 / n) h\left(X_{1}, \ldots, X_{n}\right)\right] \leq \beta(L(\omega))+H^{*} \quad \text { a.s. }
\end{gathered}
$$

where $h\left(X_{1}, \ldots, X_{n}\right)=E\left[-\ln f_{n}\left(X_{1}, \ldots, X_{n}\right)\right]$ is the differential entropy for $\left(X_{1}, \ldots, X_{n}\right)$, and

$$
\begin{aligned}
H_{*} & =\liminf _{n \rightarrow \infty}(1 / n)\left[\sum_{k=1}^{n} h\left(X_{k}\right)-h\left(X_{1}, \ldots, X_{n}\right)\right] \\
& =\liminf _{n \rightarrow \infty}(1 / n) D\left(f_{n} \| q_{n}\right), \\
H^{*} & =\limsup _{n \rightarrow \infty}(1 / n)\left[\sum_{k=1}^{n} h\left(X_{k}\right)-h\left(X_{1}, \ldots, X_{n}\right)\right] \\
& =\limsup _{n \rightarrow \infty}(1 / n) D\left(f_{n} \| q_{n}\right),
\end{aligned}
$$

where $\alpha(L(\omega)), \beta(L(\omega))$ are denoted by (3.9)-(3.13).

Corollary 3.6. If $P=Q$, or $\left\{X_{n}, n \geq 1\right\}$ are independent random variables, and there exists $s_{0}>0$, such that (2.1) holds, then

$$
\lim _{n \rightarrow \infty}\left[p_{n}(\omega)-(1 / n) h\left(X_{1}, \ldots, X_{n}\right)\right]=0 \text { a.s. }
$$

\section{Acknowledgments}

This research is supported by the National Natural Science Foundation of China (Grants nos. 10671052 and 10571008), the Natural Science Foundation of Beijing (Grant no. 1072004), Funding Project for Academic Human Resources Development in Institutions of Higher Learning Under the Jurisdiction of Beijing Municipality, the Basic Research and Frontier Technology Foundation of Henan (Grant no. 072300410090), and the Natural Science Research Project of Henan (Grant no. 2008B110009). The authors would like to thank the editor and the referees for helpful comments, which helped to improve an earlier version of the paper.

\section{References}

[1] R. G. Laha and V. K. Rohatgi, Probability Theory, Wiley Series in Probability and Mathematical Statistic, John Wiley \& Sons, New York, NY, USA, 1979.

[2] P. Billingsley, Probability and Measure, Wiley Series in Probability and Mathematical Statistics, John Wiley \& Sons, New York, NY, USA, 2nd edition, 1986.

[3] W. Liu, Relative entropy densities and a class of limit theorems of the sequence of $m$-valued random variables, The Annals of Probability, vol. 18, no. 2, pp. 829839, 1990.

[4] W. Liu, A class of strong deviation theorems and Laplace transform methods, Chinese Science Bulletin, vol. 43, no. 10, pp. 10361041, 1998.

[5] W. Liu and Y. Wang, A strong limit theorem expressed by inequalities for the sequences of absolutely continuous random variables, Hiroshima Mathematical Journal, vol. 32, no. 3, pp. 379387, 2002.

[6] W. Yang, Some limit properties for Markov chains indexed by a homogeneous tree, Statistics $\mathcal{E}$ Probability Letters, vol. 65, no. 3, pp. 241250, 2003. 
[7] W. Liu, Strong Deviation Theorems and Analytic Method, Science Press, Beijing, China, 2003.

[8] J. L. Doob, Stochastic Processes, John Wiley \& Sons, New York, NY, USA, 1953.

[9] W. Liu and J. Wang, A strong limit theorem on gambling systems, Journal of Multivariate Analysis, vol. 84, no. 2, pp. 262273, 2003.

[10] T. M. Cover and J. A. Thomas, Elements of Information Theory, Wiley Series in Telecommunications, John Wiley \& Sons, New York, NY, USA, 1991.

[11] C. E. Shannon, A mathematical theory of communication, The Bell System Technical Journal, vol. 27, pp. 379423, 623-656, 1948.

[12] P. H. Algoet and T. M. Cover, A sandwich proof of the Shannon-McMillan-Breiman theorem, The Annals of Probability, vol. 16, no. 2, pp. 899909, 1988.

[13] A. R. Barron, The strong ergodic theorem for densities: generalized Shannon-McMillan-Breiman theorem, The Annals of Probability, vol. 13, no. 4, pp. 12921303, 1985.

[14] K. L. Chung, A note on the ergodic theorem of information theory, The Annals of Mathematical Statistics, vol. 32, no. 2, pp. 612614, 1961.

[15] J. C. Kieffer, A simple proof of the Moy-Perez generalization of the Shannon-McMillan theorem, Pacific Journal of Mathematics, vol. 51, pp. 203206, 1974.

[16] J. C. Kieffer, A counterexample to Perez's generalization of the Shannon-McMillan theorem, The Annals of Probability, vol. 1, no. 2, pp. 362364, 1973.

[17] B. McMillan, The basic theorems of information theory, The Annals of Mathematical Statistics, vol. 24, no. 2, pp. 196219, 1953.

[18] W. Liu and W. Yang, An extension of Shannon-McMillan theorem and some limit properties for nonhomogeneous Markov chains, Stochastic Processes and Their Applications, vol. 61, no. 1, pp. 129145, 1996.

[19] W. Liu and W. Yang, The Markov approximation of the sequences of $N$-valued random variables and a class of small deviation theorems, Stochastic Processes and Their Applications, vol. 89, no. 1, pp. 117130, 2000.

[20] R. M. Gray, Entropy and Information Theory, Springer, New York, NY, USA, 1990. 


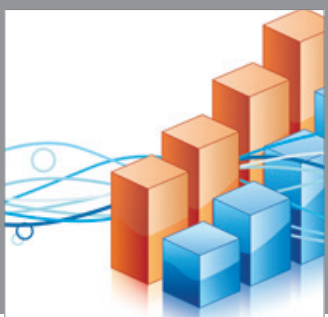

Advances in

Operations Research

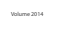

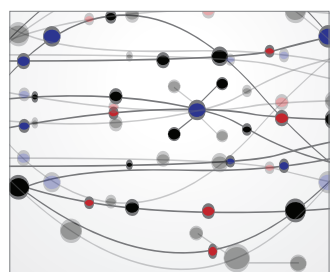

\section{The Scientific} World Journal
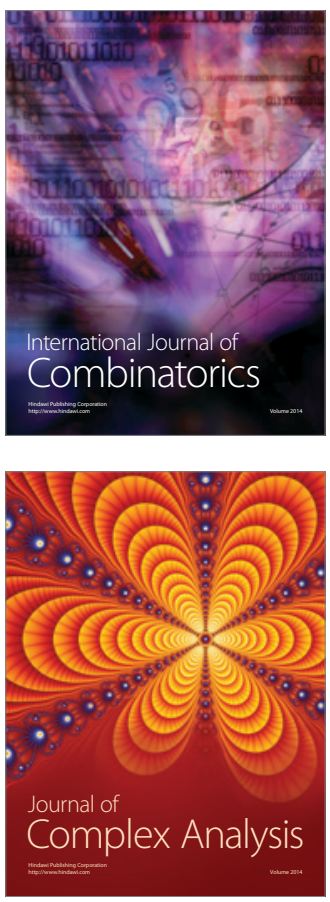

International Journal of

Mathematics and

Mathematical

Sciences
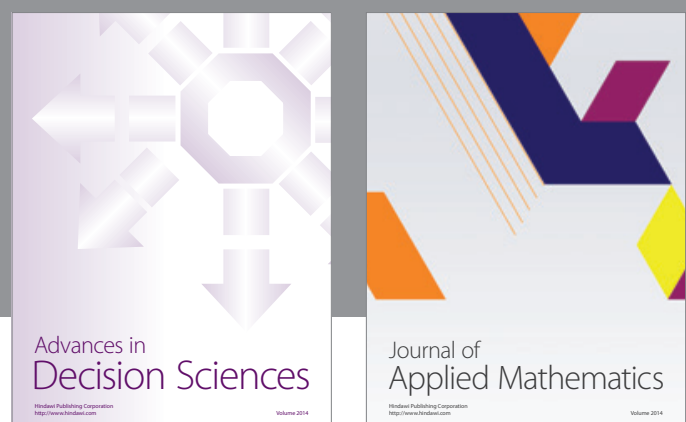

Journal of

Applied Mathematics
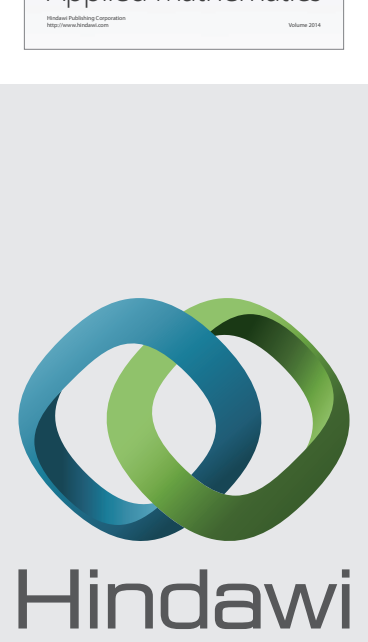

Submit your manuscripts at http://www.hindawi.com
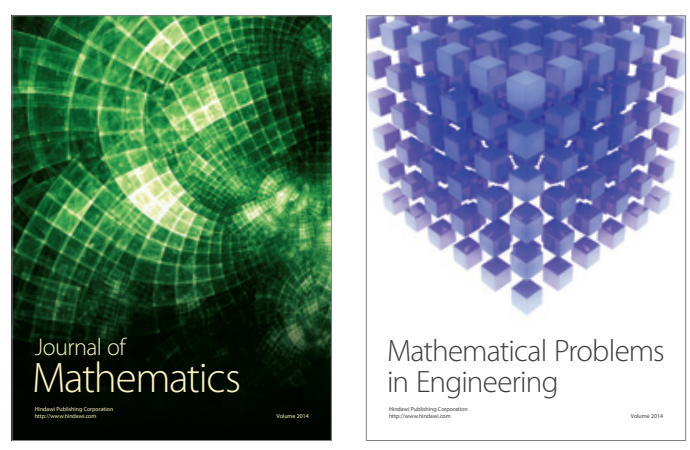

Mathematical Problems in Engineering
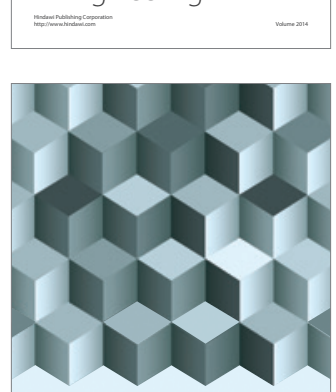

Journal of

Function Spaces
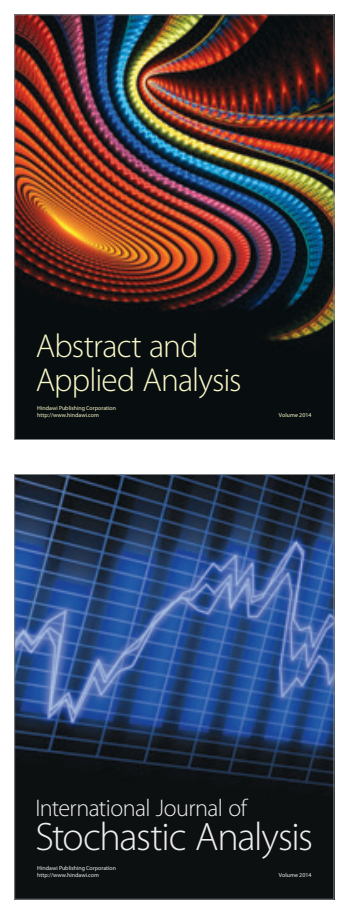

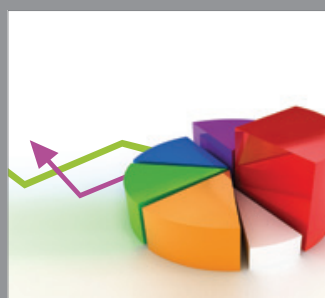

ournal of

Probability and Statistics

Promensencen
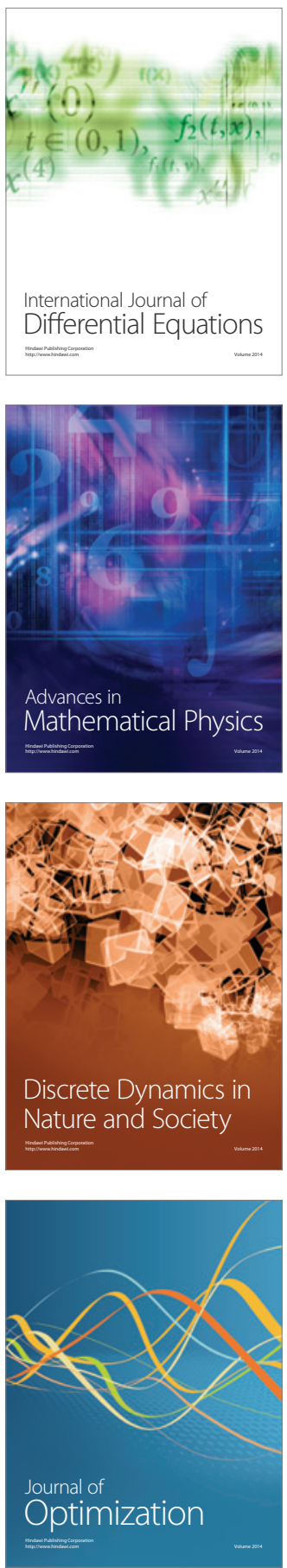